hep-ph/9211309

CPTH-A206.1192

FTUAM 92/35

IEM-FT-63/92

October 1992

\title{
Dynamical supersymmetry breaking with a large internal dimension $\mathrm{s}$
}

\author{
I. Antoniadis \\ Centre de Physique Théorique, Ecole Polytechnique, 91128 Palaiseau, France \\ C. Muñoz \\ Dept. de Física Teórica C-XI, Univ. Autónoma de Madrid, 28049 Madrid, Spain \\ M. Quirós \\ Instituto de Estructura de la Materia, Serrano 123, 28006 Madrid, Spain
}

\begin{abstract}
Supersymmetry breaking in string perturbation theory predicts the existence of a new dimension at the $\mathrm{TeV}$ scale. The simplest realization of the minimal supersymmetric Standard Model in the context of this mechanism has two important consequences: (i) A natural solution to the $\mu$-problem; (ii) The absence of quadratic divergences in the cosmological constant, which leads to a dynamical determination of the supersymmetry breaking and electroweak scale. We present an explicit example in which the whole particle spectrum is given as a function of the top quark mass. A generic prediction of this mechanism is the existence of Kaluza-Klein excitations for gauge bosons and higgses. In particular the first excitation of the photon could be accessible to future accelerators and give a clear signal of the proposed mechanism.
\end{abstract}

\footnotetext{
${ }^{1}$ Work partly supported by IN2P3-CICYT under contract PTh91-2 and by EEC contracts SC1915053 and SC1-CT92-0792
} 


\section{Introduction}

One of the main issues in string theory is the supersymmetry breaking, which constitutes a basic ingredient to connect the effective field theory at the Planck scale $M_{p}$ to the observed low energy physics. It turns out that the scale of supersymmetry breaking $m_{s}$ does not correspond to an independent parameter of the compactification in four dimensions [1]. In every consistent perturbative solution with spontaneously broken supersymmetry, $m_{s}$ is necessarily proportional to the inverse size of some internal dimension(s) $1 / R$ [2, 3]. Since $m_{s}$ is expected to be of the order of the scale of weak interactions to protect the gauge hierarchy, it follows that the decompactification scale must be in the $\mathrm{TeV}$ range.

From the field theory point of view such a large dimension would be a theoretical disaster since gauge couplings increase, and the theory becomes non-perturbative, very rapidly above $1 / R$. More serious, the effective theory is non-renormalizable in that region and decoupling is generally lost. In string theory the latter problem is automatically solved due to the finiteness property, while the former can be avoided in a large class of four-dimensional models [4]. Its solution is based on the observation that, before supersymmetry breaking, the gauge couplings in these models do not depend on the large dimension: the physical reason is that the Kaluza-Klein (KK) excitations form, at each level, $N=4$ supermultiplets which give vanishing contribution to the $\beta$-functions. The main achievement of these string models is to accomodate this property with that of chirality for the massless spectrum. After supersymmetry breaking the dependence is exponentially suppressed in the large radius limit [4. This phenomenon can be explicitely shown for orbifold compactifications. In that case the consistency of the theory after the chiral projection leads also to the existence of the so-called twisted states which have not KK-excitations.

In this class of models supersymmetry is spontaneously broken along a flat direction, corresponding to arbitrary values of $R$, and a vanishing vacuum energy at the tree-level. On the other hand, an important property of this mechanism is the absence of quadratic divergences in the cosmological constant. This allows the radia- 
tive determination of the supersymmetry breaking and electroweak scales, as in the no-scale supergravity models [5], by minimizing the full one-loop effective potential. Both mass-parameters are given in terms of a new scale, $Q_{0}$, which is dynamically generated through the running of the renormalization group equations (RGEs) and is hierarchically smaller than $M_{p}$.

In this paper, we work out in detail a simple realization of the minimal supersymmetric Standard Model (MSSM) assuming a string construction which exhibits the above properties. We find that the model is strongly constrained both by theoretical and phenomenological requirements. In particular, quarks and leptons are twisted states, while the Higgs sector plays a very special role. In fact the second Higgs doublet, characteristic of supersymmetric theories, is identified with the first KK-excitation of the first Higgs doublet carrying opposite hypercharge. This allows a natural generation of the $\mu$-parameter in the broken supersymmetric theory, given in terms of the compactification radius. The only non-vanishing soft breaking terms at the Planck scale are a common gaugino mass equal to $\mu$, as well as non-universal Higgs masses also determined by $\mu$. After imposing all minimization conditions one is left with one free parameter, the top Yukawa coupling $h_{t}$. We find two allowed regions: a light top region, where $m_{t} \lesssim 105 \mathrm{GeV}$, and a heavy top region, where $140 \mathrm{GeV} \lesssim m_{t} \lesssim 155 \mathrm{GeV}$. In both cases, the dynamically generated scale $Q_{0}$ is close to the electroweak scale. A characteristic signature of these models is the existence of KK-modes of gauge bosons and higgses at low energy. It turns out that the lightest one, an excited photon $\gamma^{*}$, could be accesible to future accelerators.

To be self-contained we review, in Section 2, the main features of orbifold compactifications that will be used, as well as the mechanism of supersymmetry breaking with a large internal dimension. This is the generalization of the Scherk-Schwarz field-theoretical method [6, 7] in string theory [8, 3]. The $\mathbf{Z}_{4}$ example is worked out for pedagogical purposes. In Section 3, we describe the dynamical determination of scales. We also compare this approach to the non-perturbative mechanism of supersymmetry breaking by gaugino condensation [9]. In Section 4, we present the simplest embedding of the MSSM in the context of this mechanism and we explain how the 
$\mu$-problem is solved. All tree-level soft breaking parameters are obtained in terms of one scale $1 / R$ and they are non-universal in the Higgs sector. In Section 5, we discuss some aspects related to the new physics of Kaluza-Klein excitations. In Section 6, we solve numerically the renormalization group equations using the above boundary conditions. We find the allowed region consistent with all theoretical and experimental requirements for the only free parameter, namely $h_{t}$. The spectra of supersymmetric particles, higgses and the lowest KK-excitation of the photon are plotted, as functions of the top-quark mass. Finally the RGEs for couplings and soft breaking parameters of the MSSM are collected, for completeness, in Appendix A.

\section{The mechanism of supersymmetry breaking}

We review here the mechanism of supersymmetry breaking with a large internal dimension in the case of orbifold compactifications of the heterotic superstring.

The physical states in orbifolds can be classified in two categories [10]:

- Untwisted states, which appear in the toroidal compactification and survive after the orbifold projection. In toroidal compactifications every state in the higher dimensional theory gives rise to a tower of states in four-dimensions with a mass squared shift equal to $1 / R^{2}$, where $R$ is the corresponding compactification radius. These are the Kaluza-Klein excitations and their mass comes from the components of the momentum, along the compactified direction, which is quantized in units of $1 / R$. In string theory there are also winding states with a mass squared shift equal to $R^{2} / 4$, in Planck units:

$$
M^{2}=M_{0}^{2}+\frac{m^{2}}{R^{2}}+\frac{n^{2} R^{2}}{4}, \quad m, n=0, \pm 1, \pm 2, \ldots,
$$

where $m$ and $n$ are the momentum and winding numbers, respectively, and $M_{0}$ stands for other $R$-independent contributions to the mass. In the large radius limit we are interested, the winding states $(n \neq 0)$ are superheavy and, thus, irrelevant for our purposes. In field theories toroidal compactifications lead always to non-chiral four-dimensional spectra. In string theory, orbifolds provide 
a simple way of obtaining chirality. The states from the toroidal compactification are projected into the subset of states which are invariant under the discrete orbifold group. This projection leads to a chiral massless spectrum with $N=1$ supersymmetry.

- Twisted states, which are necessary for the internal consistency of the theory and correspond to string excitations around the fixed points of the torus under the orbifold group. Their masses do not depend on the radius of the torus and, therefore, they do not have KK-excitations. These states are characteristic of string theory and do not have any analog in field theories.

When compactifying the ten-dimensional theory to four dimensions there appear three complex internal planes. In a general $N=1$ supersymmetric theory the Hilbert space of states can be divided into three sectors depending on the transformation properties of the internal planes under the action of the orbifold group.

1. The $N=4$ sector, where all three planes are untwisted, which can be seen as the truncation of a theory with $N=4$ supersymmetry. The masses of the corresponding states depend on the deformations of all complex planes, in particular on all internal radii. Its contribution to threshold corrections vanishes due to the underlying $N=4$ symmetry 11$]$.

2. $N=2$ sectors, where one plane is untwisted and two planes are twisted, which can be seen as the truncation of a theory with $N=2$ supersymmetry. The masses now depend only on the deformations of the untwisted plane. As a consequence threshold corrections can depend only on those deformations [12].

3. $N=1$ sectors, where all planes are twisted. The corresponding states and threshold corrections do not depend on any deformation of the internal planes.

Let us consider for simplicity only one of the three complex internal planes with a large compactification radius $R$. To avoid troubles with perturbation theory gauge couplings should not depend on $R$. The simplest way is to choose a plane which is twisted in all $N=2$ sectors. 
To break spontaneously supersymmetry we use the Scherk-Schwarz mechanism [6] that makes use of a global, or local, continuous symmetry of the higher dimensional theory. Upon compactification of the coordinate $X$ on a circle of radius $R$ the higher dimensional fields can be choosen to be periodic up to a symmetry transformation:

$$
X \rightarrow X+2 \pi R, \quad \Phi_{q} \rightarrow e^{2 i \pi q \omega} \Phi_{q},
$$

where $q$ is the charge of the field $\Phi_{q}$ and $\omega$ is an arbitrary parameter of the transformation. The boundary condition (2.2) leads to a shift in the momentum $P$ along the $X$ direction $q \omega / R$, i.e.

$$
P=\frac{m}{R}+\frac{q \omega}{R}, \quad m=0, \pm 1, \pm 2, \ldots,
$$

which in turn leads to a mass shift in the four-dimensional theory. If the gravitino field has a non-zero charge $q_{3 / 2}$, supersymmetry is broken at an arbitrary scale $q_{3 / 2} \omega / R$.

In theories with no continuous symmetries (left over after compactification) transforming the gravitino field, as in the case of string theory, the above mechanism still works for a discrete symmetry [7]. In that case the parameter $\omega$ is quantized and the scale of supersymmetry breaking is determined only by $R$. Note that, although supersymmetry is broken, $R$ is undetermined at the tree level. For the case of a $\mathbf{Z}_{N}$ symmetry, $\omega=1$ and the charges $q$ are multiples of $1 / N$. This mechanism was extended to string theory in Ref. [8, 3]. Equations (2.2) and (2.3) remain valid for all states with zero winding number. Consistency of the theory also requires that the symmetry generator $q$ must have the same transformation properties as the coordinate $X$ under the action of the orbifold group. As a result, non-zero mass shifts (2.3) appear only in sectors where $X$ is untwisted.

To illustrate the above ideas we present a simple example based on the $\mathbf{Z}_{4}$ orbifold 四. The corresponding orbifold group acting on the three complex coordinates $\left(X_{1}, X_{2}, X_{3}\right)$, and their 2D left-moving fermionic superpartners $\left(\Psi_{1}, \Psi_{2}, \Psi_{3}\right)$, is generated by the element $g=(i, i,-1)\left(g^{4}=1\right)$. The only $N=2$ sector corresponds to the element $g^{2}=(-1,-1,1)$ which leaves the third internal plane invariant. The first and second planes are therefore good candidates to have large compactification radii. 
The generator of a space-time symmetry with the desired properties corresponds on the world-sheet to the following left-moving complex $U(1)$ current:

$$
J=\frac{1}{\sqrt{2}}\left(\Psi_{1} \psi+\bar{\Psi}_{1} \operatorname{Re} \Psi_{3}\right)
$$

where $\psi^{\mu}$ are the left-moving 2D superpartners of the 4D space-time coordinates $x^{\mu}$; $\psi$ denotes any of the components of $\psi^{\mu}$. The current (2.4) does not commute with the world-sheet supercurrent $T_{F}$

$$
T_{F}=\psi^{\mu} \partial x_{\mu}+\frac{1}{2} \sum_{i=1}^{3}\left(\Psi^{i} \partial \bar{X}_{i}+\text { h.c. }\right) \text {. }
$$

The only allowed discrete transformations that commute with $T_{F}$ are $e^{2 i \pi} \oint J$, which leave the $2 \mathrm{D}$ fermions $\Psi_{1}, \psi, \operatorname{Re} \Psi_{3}$ invariant. Now we concentrate in the $N=4$ sector, which is the only one where the coordinate $X_{1}\left(\right.$ or $\left.X_{2}\right)$ is untwisted and therefore we can have non-zero mass shifts. It is easy to show that the above transformation acts on physical states as a $\mathbf{Z}_{2}$ space-time parity, namely $(-1)^{2 s}$, where $s$ is the spin. In fact, bosons come from the Neveu-Schwarz sector where 2D fermions are anti-periodic and remain invariant under the transformation $e^{2 i \pi} \oint J$. On the other hand, fermions come from the Ramond sector where 2D fermions are periodic and the physical states, transforming in the spinorial representation, change sign. As a result, the only states which receive mass shifts from the supersymmetry breaking mechanism are the fermions in the $N=4$ sector which survive the orbifold projection. These are the gravitino, gauginos and all fermions in matter supermultiplets (and their KKexcitations). Their mass is provided by (2.3) with $\omega=1$ and $q=\frac{1}{2}$. The general mass formula is:

$$
M^{2}=M_{0}^{2}+\frac{\left(m+\frac{1-n}{2}\right)^{2}}{R^{2}}+\frac{n^{2} R^{2}}{4}, \quad m, n=0, \pm 1, \pm 2, \ldots,
$$

where we also included the winding number $n$ for completeness. As in (2.1) $M_{0}$ denotes a possible contribution to the mass with a different origin, e.g. vacuum expectation values at some scale.

All fermions in the $N=4$ sector which live in non-chiral supermultiplets get a Majorana mass (2.6). The mass of fermions which belong to chiral supermultiplets 
looks puzzling and requires an explanation. In fact, the supersymmetry breaking mechanism changes the complexification of fermions within the infinite tower of KKexcitations: the one corresponding to the momentum number $m$ is complexified with that corresponding to $-m-1$, as can be seen from (2.6), and becomes a Dirac fermion. In particular the massless chiral fermion with $m=0$ is complexified with the (antichiral) fermion with $m=-1$ from the first KK-excitation and gets a Dirac mass equal to $1 / 2 R$. From the point of view of the effective field theory this phenomenon is described by supersymmetric masses in the superpotential,

$$
W=\frac{1}{2 R} \Phi_{0} \Phi_{-1}
$$

together with appropriate soft breaking terms for the scalar components, such that their final masses remain unchanged:

$$
V_{\mathrm{sb}}=-\frac{1}{4 R^{2}}\left|\Phi_{0}\right|^{2}+\frac{3}{4 R^{2}}\left|\Phi_{-1}\right|^{2}
$$

where $\Phi_{m}$ denotes the $m$-th KK-excitation, and we are using the same symbols for superfields and their scalar components. The superpotential (2.7) leads to a fermion mass $1 / 2 R$, while (2.7) and (2.8) lead to scalar masses equal to zero for $\Phi_{0}$ and to $1 / R$ for $\Phi_{-1}$. A general consequence of the pattern of supersymmetry breaking is that, in realistic models, quark and lepton multiplets should belong to twisted sectors. Otherwise they would become massive. Therefore these matter fields do not have light KK-excitations.

Once supersymmetry is broken gauge couplings in general acquire a dependence on $R$ through radiative corrections which could bring us back to the problem of spoiling the perturbative expansion. However the corrections are expected to be suppressed by the supersymmetry breaking scale, proportional to $1 / R$. In fact they are exponentially suppressed (up to constants) [4]. Moreover the radius dependence of the one-loop cosmological constant can be calculated with the result [13, 4]:

$$
\Lambda_{\mathrm{cosm}} \sim\left(n_{B}-n_{F}\right) \frac{1}{R^{4}}+\ldots,
$$

where $n_{B}$ and $n_{F}$ are the number of massless bosons and fermions, respectively, after the supersymmetry breaking, and the ellipsis stands for corrections which are 
exponentially suppressed in terms of $R^{2}$. The absence of $1 / R^{2}$ terms in (2.9) implies the vanishing of $\operatorname{Str}_{\mathcal{M}}$ and, hence, of quadratic divergences in the cosmological constant. The $1 / R^{4}$ suppression is similar to the one obtained in $N=4$ supergravity and is a consequence of having broken supersymmetry only in the $N=4$ sector.

Although the above discussion dealt with the $\mathbf{Z}_{4}$ orbifold, the results apply to a more general class of models [4]. In fact, whenever we choose a radius corresponding to a plane untwisted only in the $N=4$ sector, the pattern of supersymmetry breaking will be the same as described above, unless the discrete symmetry group is different from $\mathbf{Z}_{2}$. On the other hand, there exists the possibility of choosing a plane untwisted even in some $N=2$ sectors in models where threshold corrections do not depend on the corresponding radius. In that case the scalar masses from those $N=2$ sectors are shifted by the supersymmetry breaking mechanism by the same amount as in (2.6). This possibility is model dependent and will not be considered in our analysis.

\section{Dynamical determination of scales}

The mechanism of supersymmetry breaking above described yields an $N=1$ supersymmetric effective field theory with soft breaking terms coming from the spontaneous breaking of supergravity. All these terms are proportional to the inverse size $1 / R$ of the internal dimension. The soft breaking terms can trigger radiative gauge symmetry breaking [14] at a scale close to the supersymmetry breaking scale $m_{s} \sim 1 / R$ when a Higgs field acquires a non-zero vacuum expectation value (VEV) $v$. This in general happens if there is some large Yukawa coupling $h$ in the theory, which drives the mass squared of the Higgs to negative values. The value of $v$ is proportional to $m_{s}$, which is the only scale appearing in the renormalizable field theory as a consequence of the absence of quadratic divergences in the scalar masses. Note that $R$, and so $m_{s}$, is arbitrary at the string tree-level since it is given by the VEV of a so-called modulus field $T$ which remains a flat direction at this level after the supersymmetry breaking, while the cosmological constant vanishes.

This situation is reminiscent of the no-scale models in $N=1$ supergravity [5]. 
One expects that, once supersymmetry is broken, string radiative corrections will lift the flatness by generating a non trivial potential for the field $T$. Minimization of this potential with respect to the Higgs field and $T$ will fix $v$ and $R$ in terms of $M_{p}$ (the only scale in the string theory). In general one would expect both scales to be of the order of magnitude of $M_{p}$. However, in the absence of quadratic divergences in the cosmological constant, there is no $M_{p}^{2} T^{2}$ term in the effective potential. So in the limit $M_{p} \rightarrow \infty$ only the renormalizable part of the potential survives and a new scale $Q_{0}$ is dynamically generated through the running of the renormalization group equations. Examples of this mechanism have been analyzed in the past, in the context of no-scale models [5], and the scale $Q_{0}$ was found to be

$$
Q_{0} \sim M_{p} e^{-\frac{4 \pi}{h^{2}\left(M_{p}\right)} \mathcal{O}(1)}
$$

where $M_{p}$ plays here the role of the scale fixing the boundary conditions. Note that $Q_{0}$ is RGE invariant and, hence, a new physical scale depending only on the value of the Yukawa coupling at $M_{p}$. If $h$ is not too large, $Q_{0}$ can be hierarchically smaller than $M_{p}$. Both $v$ and $m_{s}$ are then proportional and of the order of magnitude of $Q_{0}$; this could explain dynamically the hierarchy between the weak and Planck scales without any fine-tuning.

The class of string models we have introduced in the previous section provides good candidates for a dynamical generation of the $Q_{0}$ scale, since the condition for the vanishing of $\operatorname{Str}_{\mathcal{M}}{ }^{2}$ is fulfilled as can be seen from (2.9). We stress that the determination of $Q_{0}$ has a perturbative nature since it relies upon the one-loop effective potential which is calculable in string theory. This is in contrast with non-perturbative mechanisms of supersymmetry breaking, e.g. gaugino condensation in the hidden sector [9]. In the latter, there is a non-perturbatively generated condensation scale $\Lambda$ which triggers a supersymmetry breaking scale in the observable sector through gravitational interactions $m_{s} \sim \Lambda^{3} / M_{p}^{2}$. This method, since it relies on non-perturbative effects, suffers from a partial lack of calculability at the present state of the art. The situation becomes worse in the context of string theory, where there is no known framework of studying non-perturbative phenomena. Another important difference 
between the two mechanisms is that the value of $Q_{0}$ is controled by parameters that can be measured at low energy, while $\Lambda$ is controled by the hidden sector of the theory, which cannot be directly measured by present experiments.

In 4D strings all coupling constants depend on the VEV's of scalar fields, the moduli, which determine the shape and size of the internal space. Their values should be determined dynamically by the minimization of the effective potential. As long as supersymmetry remains unbroken they are undetermined since the moduli are exact flat directions (in perturbation theory). Once supersymmetry is broken the degeneracy is lifted by the generated potential. An important property of string theory, in the absence of supersymmetry breaking, is the duality symmetry which relates small and large radii by exchanging momentum and winding modes. In the simplest case of one radius, as can be seen from (2.1), the spectrum is invariant under $R \rightarrow \frac{2}{R}$ and $n \leftrightarrow m$. This symmetry is expected to remain at the level of the effective field theory of massless modes $(m=n=0)$ even in the presence of gaugino condensation. This implies that self-dual points, e.g. $R=\sqrt{2}$, are always extrema of the potential, and possibly minima unless duality is spontaneously broken. This result does not necessarily requires that gaugino condensation breaks supersymmetry. If supersymmetry is broken perturbatively by a large radius a modified duality transformation still remains as an invariance of the string theory. As can be seen from (2.6) the shifted spectrum is invariant under $R \rightarrow \frac{1}{R}$ and $n \leftrightarrow(2 m+1-n)$. In this case the effective field theory of originally massless states is not any longer duality invariant. For instance, under this transformation the gauginos $(m=n=0)$ are related to superheavy winding states $(m=0, n=1)$ which are not included in the effective theory. As a result the self-dual point $R=1$ is not in general an extremum of the potential and, as we discussed before, $R$ can be fixed at a very large value related to a new dynamical scale $Q_{0}$. Radiative corrections in the supersymmetry broken theory are expected to generate also a potential for all other moduli unrelated to $R$, lifting their degeneracy. For them the effective theory remains invariant under the corresponding duality transformations and self-dual points will be extrema of the potential.

In addition to the moduli there is a universal scalar field, the dilaton, which plays 
a very particular role because its VEV determines the tree-level 4D string coupling constant. It is clear that the $\mathrm{VEV}$ of this field cannot be fixed at any given order of string perturbation theory, unless some resummation could be performed. The situation is not improved even in the presence of one-gaugino condensation, where a runaway potential is obtained for the dilaton. This problem can be fixed if two different condensates are present [15]. A possible scenario would be fixing the dilaton and moduli which appear in the gauge couplings by such non-perturbative effects without breaking supersymmetry, while supersymmetry is broken by some of the remaining radii using the perturbative approach described above.

\section{The minimal embedding of the Standard Model and a solution to the $\mu$-problem}

In this section we present the effective theory corresponding to the simplest embedding of the minimal supersymmetric Standard Model in the general context described above.

As we already pointed out in Section 2, all gauginos get a common Majorana mass given by (2.6),

$$
M_{1 / 2}=\frac{1}{2 R}
$$

since they belong to non-chiral supermultiplets from the untwisted sector. On the other hand quarks and leptons should be identified with twisted states, having no light KK-excitations. Thus, all their corresponding soft breaking scalar masses vanish,

$$
m_{\tilde{q}}=m_{\tilde{\ell}}=0
$$

In the MSSM one needs a Higgs sector with two doublets, $H_{1}$ coupled to down quarks and leptons, and $H_{2}$ coupled to up quarks. These couplings exhibit a global Peccei-Quinn symmetry, which is spontaneously broken along with the electroweak symmetry, giving rise to a massless axion. To avoid this problem one has to introduce a supersymmetric mass-term in the superpotential $\mu H_{1} H_{2}$, where $\mu$ must be of the 
order of the electroweak scale. The introduction of such a parameter looks completely unnatural, since it brings back the hierarchy problem at tree-level even for unbroken supersymmetry. Its origin requires an explanation at the more fundamental level. In fact, if the higgses belong to the untwisted sector, the mechanism of supersymmetry breaking we described above provides a natural solution to the $\mu$ problem. Following Section 2, one can identify one of the higgses with the first KK-excitation of the other. Then the Higgs superpotential and soft breaking mass terms are given by (2.7) and (2.8). As a consequence, one obtains

$$
\mu=\frac{1}{2 R}
$$

Depending on which Higgs field is assigned to the first KK-excitation, one obtains two distinct cases of Higgs masses:

$$
\begin{array}{llllll}
\text { case } 1: & H_{1}=\Phi_{-1} & H_{2}=\Phi_{0} & \Rightarrow & m_{1}=\frac{1}{R} & m_{2}=0 \\
\text { case 2 : } & H_{2}=\Phi_{-1} & H_{1}=\Phi_{0} & \Rightarrow & m_{1}=0 & m_{2}=\frac{1}{R} .
\end{array}
$$

Note that in (2.8) there is no soft breaking mixing between the two higgses, $B \mu \Phi_{0} \Phi_{-1}$, implying that the soft breaking parameter $B$ vanishes,

$$
B=0
$$

In both cases (4.4) we use only one Higgs doublet that was massless before supersymmetry breaking. This raises the problem of anomaly cancellation in the supersymmetric theory. Since all massless fermions from the untwisted sector become massive in the broken theory, while those from the twisted sectors remain massless, the anomaly cancellation condition after supersymmetry breaking only involves the twisted states. Therefore, there are two possible scenarios consistent at the string level:

1. The supersymmetric theory is anomaly free, implying that $U(1)_{Y}$-hypercharge anomalies must cancel separately in untwisted and twisted sectors. In this case there should exist extra untwisted matter representations. The simplest possibility consists in having an additional Higgs doublet with no coupling to quarks and leptons. 
2. The supersymmetric theory has only one massless Higgs doublet and the $U(1)_{Y}$ is anomalous. As all $S U(2)$ and gravitational mixed anomalies are proportional to $\operatorname{Tr} Y$, this anomaly can be cancelled by a Green-Schwarz counterterm [16]. Although the supersymmetric theory appears to be unrealistic since the hypercharge would be broken at the Planck scale, in the broken theory the $U(1)_{Y}$ becomes anomaly free and one is left with the minimal supersymmetric standard model. This unconventional possibility could be interesting for model building.

It remains to study the $A$ soft breaking parameters, which correspond to the trilinear couplings in the scalar potential. They are given by the on-shell tree-level amplitude involving three complex scalars whose fermionic partners have the same chirality. This amplitude vanishes as in the supersymmetric theory, since the scalar vertex operators remain unchanged after supersymmetry breaking. Therefore

$$
A=0 .
$$

To summarize, the simplest embedding of the MSSM we have just described leads to the tree-level values of masses and soft-breaking parameters given in (4.1-4.6). They are non-universal in the Higgs sector (4.4), and they are given in terms of one mass scale, namely the inverse size of the extra dimension $1 / R$. The latter corresponds to the VEV of the modulus field $T$ which is a flat direction of the tree-level potential. As we explained in Section 3, the value of $1 / R$ along with the VEVs of the two Higgs doublets should be dynamically determined by minimizing the one-loop effective potential with respect to these fields. In the leading logarithmic approximation, this is equivalent to considering the renormalization group equations of the MSSM with boundary conditions defined by (4.1-4.6). The resulting VEVs will then be proportional to the RGE-invariant scale $Q_{0}$, defined in (3.1) as the scale where the determinant of the Higgs mass-squared matrix becomes negative and triggers electroweak symmetry breaking:

$$
\operatorname{det} \mathcal{M}_{H}^{2}\left(Q_{0}\right)=0 \text {. }
$$

As a consequence of the vanishing of all quadratic divergences, $Q_{0}$ is independent 
of $M_{p}$ and can be hierarchically smaller depending on the largest Yukawa coupling, namely that of the top quark $h_{t}$. Note that $Q_{0}$ is also independent of the supersymmetry breaking scale $1 / R$. The minimization of the effective potential with respect to the Higgs fields $H_{1}$ and $H_{2}$ fixes their VEVs in terms of $1 / R$, while the minimization with respect to $T$ fixes $1 / R$ in terms of $Q_{0}$. As a result, all low-energy masses are fixed in terms of $h_{t}$. Any particular string model which provides a definite prediction for $h_{t}$ will lead to a precise prediction for the low-energy mass spectrum of the theory. Here, since we are not dealing with a particular model, we will consider $h_{t}$ as a free parameter, and we will replace the minimization with respect to $T$ by the phenomenological condition on the value of the $Z$-mass. In Section 6 the mass-spectrum will be derived as a function of the top-quark mass $m_{t}$. As a consistency check of the procedure, one should verify that the obtained value for $Q_{0}$ is of the order of the scale of weak interactions.

\section{The physics of Kaluza-Klein excitations}

An unavoidable consequence of the proposed mechanism of supersymmetry breaking with a large internal dimension is the existence of KK-excitations at low energy. The lightest of such states is the first excitation of the photon, $\gamma^{*}$, with a mass

$$
M_{\gamma^{*}}=\frac{1}{R}
$$

accessible to future accelerators, and a very clear signal in the $\ell^{+} \ell^{-}$channel. Here, we will discuss a few theoretical aspects of their interactions, as well as possible effects and constraints at present energies that could lead to bounds or signatures of them.

As we have seen in the previous sections, only the untwisted states have KKexcitations, namely all gauge boson and Higgs supermultiplets. Although, by construction, these KK-modes do not affect the running of coupling constants, they could lead to observable effects through non-renormalizable interactions. In a supersymmetric theory, such higher dimensional interactions may come from $F$ or $D$-terms. Since quarks and leptons are in the twisted sector, the interactions corresponding 
to $F$-terms involve more than three twisted fields and, thus, are exponentially suppressed in the large radius limit [17]. This does not apply to $D$-terms which can lead to dimension six effective operators corresponding, for instance, to the exchange of massive vector bosons.

In the $N=4$ supersymmetric theory obtained from a toroidal compactification, all KK-excitations have the same quantum numbers as those of the lowest lying massless states. At the massless level, some quantum numbers are projected out because they are not invariant under the orbifold group. However, they all appear at the massive level because one can always construct linear combinations of KK-modes which are invariant under the orbifold group, and carry these quantum numbers. A simple example of this phenomenon is the hypercharge in the Higgs sector discussed in Section 4: the massive excitations of one Higgs doublet contain doublets with the opposite hypercharge. As a result, in a general $N=1$ theory, KK-excitations may have additional quantum numbers compared to those of the lowest lying massless states. This might give rise to unwanted, phenomenologically suppressed processes, as fast proton decay. However, they can be easily avoided if the model has no massless color triplets at the $N=4$ level, i.e. before the orbifold projection is applied.

Now, we concentrate on model independent dimension six operators which are produced by the exchange of KK-modes with the same quantum numbers as the lowest lying states $\left(e . g . \gamma^{*}, Z^{*}, \ldots\right)$. Either they modify the effective parameters of the Standard Model (e.g. $\left.Z^{*}, W^{*}\right)$, or they produce new effective interactions (e.g. $\gamma^{*}$, excited gluon). In the former case, the mass of the excited modes is bounded by the precision measurements of weak interactions. In the latter, the mass is constrained by bounds on new physics, obtained from those on compositeness. The general form of such operators can be written as:

$$
\frac{e^{2}}{2 M^{2}}\left(\bar{\psi} \gamma^{\mu} \psi\right)^{2},
$$

where $M$ is the mass of the intermediate state and $e$ its coupling to the massless fermions $\psi$ at low energy. The tree-level coupling of a massive KK-mode, corresponding to the momentum number $m$ (see (2.1)), to two massless twisted states is 
proportional to $\delta^{-m^{2} / 2 R^{2}}$, where $\delta$ is a number depending on the orbifold twist 18. In the large radius limit, this coefficient is equal to one, and the coupling is independent of $m$ and equal to the corresponding coupling of the lowest lying massless state, $m=0$. To determine $e$ in (5.2) at low energy, we need also to study the infrared running of the coupling of the massive KK-mode from the Planck scale to its mass. In this region, this mode can be treated as massless and its vertex coincides with that of the corresponding massless state. Therefore, the one loop string diagrams will have the same infrared divergence and both couplings will be renormalized in the same way.

To extract a first estimate on present bounds on the size of the internal dimension in the above context, we will use the experimental limits on the strength of fourfermion interactions [19]. The stronger bound comes from ee $\mu \mu$ which gives:

$$
R^{-1} \gtrsim \frac{\sqrt{6}}{\pi} \frac{1}{2.2} \alpha_{\mathrm{em}}^{1 / 2} \Lambda_{е е \mu \mu},
$$

where $\Lambda_{e е \mu \mu}$ is the compositeness scale. The first factor in the r.h.s. of (5.3) comes from the sum over the infinite tower of massive photons, while the second factor comes

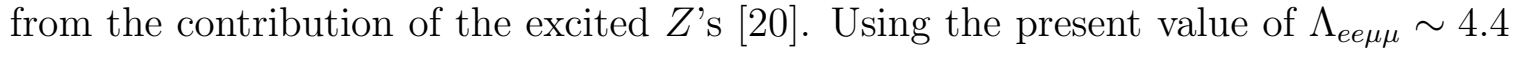
$\mathrm{TeV}$ [19], one obtains $R^{-1}>140 \mathrm{GeV}$. This means that $\gamma^{*}$ could still be detected even at LEP-200!

A number of interesting theoretical questions is related to the physics of KKmodes at high energies. As the energy increases above the decompactification scale, one produces more states which behave effectively as massless vector particles. At the field theory level, a higher gauge symmetry is required to describe consistently their interactions, and a Higgs mechanism is needed to explain the origin of their masses. This symmetry should be a small part of the full string symmetry, which is infinite dimensional in the limit of Planckian energies [21]. In this sense, the detection of these particles and the understanding of their interactions could be considered as a window to uncover the complicated underlying string structure. A related issue is the possibility of observation of the extra dimension in a reaction where some outgoing particles could "propagate" in the decompactified space. This naive field-theoretical 
picture is incorrect because there is no conserved quantum number associated to this dimension, even approximately. In fact, as we discussed, all KK-modes are unstable and decay to massless states from the twisted sector. This is a stringy phenomenon arising from the chiral character of the theory and implying that space-time behaves, below the Planck scale, always as four-dimensional from the observational point of view.

\section{The low energy particle spectrum}

Let us analyze now the radiative breaking of the $S U(2) \times U(1)_{Y}$ symmetry 14 triggered by the mechanism of spontaneous supersymmetry breaking described above. A main difference with respect to the usual low-energy supergravity models is the presence of the infinite tower of massive excitations. However, as we already pointed out, these modes do not affect the evolution of gauge couplings because they form $N=4$ multiplets. For the same reason their contribution to wave function renormalization of Higgs and matter multiplets also vanishes. On the other hand, since heavier Higgs excitations have the same coupling to matter as the lightest one, they cannot get any VEV and, thus, they do not affect the minimization of the effective potential. Therefore, all higher KK-modes can be neglected in the evolution of RGEs. Our analysis will then be similar to the usual one after taking into account the (non-universal) boundary conditions (4.1-4.6) we found in Section 4.

For completeness, we give in Appendix A the RGEs for all the parameters of the MSSM [22, 23]. Note that in these equations there is a contribution to the scalar masses coming from the $U(1)_{Y} D$-term parametrized in the general case by:

$$
S=\sum_{r} d(r) Y_{r} m_{r}^{2},
$$

where $d(r)$ is the dimension of the representation $r$. The evolution of $S$ is given by [23]:

$$
\frac{d S}{d t}=-\frac{\alpha_{1}}{4 \pi}\left(\sum_{r} d(r) Y_{r}^{2}\right) S,
$$


implying that if $S$ vanishes at some scale, it vanishes everywhere. For the case of universal boundary conditions for the scalar masses, $S=0$. In our case, $S$ seems to be non-vanishing at the string scale due to the non-universality of boundary conditions in the Higgs sector (4.4). However, this is an artifact of our truncation: taking into account the contribution to $S$ of the tower of KK-excitations, one finds at each level an equal number of massive scalars with opposite hypercharges except at the lowest level which is massless. This implies that $S$ is in fact vanishing at the string scale.

Neglecting all Yukawa couplings except the one of the top, we have in principle two free parameters at the string scale:

$$
h_{t}, \mu
$$

In order to obtain the $S U(2) \times U(1)_{Y}$ breaking at the right scale we must impose

$$
M_{Z}^{2}=\frac{1}{2}\left(g_{2}^{2}+g_{1}^{2}\right)\left(v_{1}^{2}+v_{2}^{2}\right)
$$

where $g_{2}$ and $g_{1}$ are the $S U(2)$ and $U(1)_{Y}$ gauge couplings, respectively, and $v_{1,2} \equiv$ $<H_{1,2}>$. In this way, we are left with only one free parameter and the supersymmetric spectrum will be strongly constrained.

The relevant Higgs scalar potential along the neutral direction is

$$
V\left(H_{1}, H_{2}\right)=\frac{1}{8}\left(g_{2}^{2}+g_{1}^{2}\right)\left(\left|H_{1}\right|^{2}-\left|H_{2}\right|^{2}\right)^{2}+m_{1}^{2}\left|H_{1}\right|^{2}+m_{2}^{2}\left|H_{2}\right|^{2}-m_{3}^{2}\left(H_{1} H_{2}+\text { h.c. }\right)
$$

where $m_{1}, m_{2}, m_{3}$ are related to the soft breaking parameters via expressions coming from integrating the corresponding RGEs. After integration, all renormalized soft parameters are proportional to $\mu$. In particular,

$$
\begin{array}{ll}
m_{1}^{2}=(l(t)+g(t)+a) \mu^{2} & \equiv C_{1} \mu^{2} \\
m_{2}^{2}=(l(t)+e(t)+b) \mu^{2} & \equiv C_{2} \mu^{2} \\
m_{3}^{2}=r(t) \mu^{2} & \equiv C_{3} \mu^{2},
\end{array}
$$

where the functions $l(t), g(t), e(t)$ and $r(t)$ can be found in [24]. They depend on the parameter $t=2 \log \left(M_{S U} / M_{Z}\right)$, where $M_{S U}$ is the string unification scale, as well as on $h_{t}$ and the gauge coupling constants $\alpha_{i}$. The constants $a$ and $b$ appearing in (6.6) 
are $a=3(-1), b=-1(3)$ for the case 1 (case 2 ). The minimization conditions of the scalar potential (6.5) together with the constraint (6.4) give:

$$
\begin{aligned}
\omega^{2} & =\frac{m_{1}^{2}+\frac{1}{2} M_{Z}^{2}}{m_{2}^{2}+\frac{1}{2} M_{Z}^{2}}, \\
\frac{\omega}{\omega^{2}+1} & =\frac{m_{3}^{2}}{m_{1}^{2}+m_{2}^{2}},
\end{aligned}
$$

with $\omega \equiv \frac{v_{1}}{v_{2}}$. Combining $(\underline{6.7})$ and (6.8), and using (6.6), we determine the value of $\mu$ :

$$
\mu=\left\{\frac{M_{Z}^{2}\left[\left(C_{1}+C_{2}\right)^{2}-4 C_{3}^{2} \pm\left(C_{1}-C_{2}\right) \sqrt{\left(C_{1}+C_{2}\right)^{2}-4 C_{3}^{2}}\right]}{4\left(C_{1}+C_{2}\right)\left(C_{3}^{2}-C_{1} C_{2}\right)}\right\}^{\frac{1}{2}} .
$$

It turns out that for the boundary conditions we use, the solution corresponding to the lower sign in (6.9) is unrealistic because it gives rise to imaginary values for $\mu$. We are left with only one free parameter $h_{t}$ and the whole supersymmetric mass spectrum can be obtained as a function of the top mass $m_{t}$.

We have solved numerically the RGEs of Appendix A in the approximation $h_{b}$, $h_{\tau} \ll h_{t}$ and computed the supersymmetric particle masses by means of the usual formulae [14]. All the masses are evaluated at the $M_{Z}$ scale and the running is done from $M_{S U} \simeq 0.5 \times g \times 10^{18} \mathrm{GeV}$ [25], where $g \simeq 1 / \sqrt{2}$ is the corresponding value of the string coupling constant 2 . The current experimental values that we use are:

$$
M_{Z}=91.175, \quad \alpha_{3}^{-1}\left(M_{Z}\right)=8, \quad \alpha_{\mathrm{em}}^{-1}\left(M_{Z}\right)=127.9, \quad \sin ^{2} \theta_{W}\left(M_{Z}\right)=0.2303 .
$$

$\alpha_{i}$ at $M_{S U}$ should be obtained by inserting $\alpha_{i}\left(M_{Z}\right)$ in the corresponding RGEs.

The numerical results are summarized in figs. 1 and 2, corresponding to the boundary conditions of cases 1 and 2 in (4.4), respectively, where the whole spectrum is plotted as a function of $m_{t}$. It is worth noticing that although we have only one free parameter, there are still solutions consistent with the experimental bounds. This is certainly non-trivial and the allowed solutions strongly constrain the range of the top quark mass. In fact, case 1 leads to a light top in the range

$$
90 \mathrm{GeV}<m_{t} \lesssim 105 \mathrm{GeV}
$$

\footnotetext{
${ }^{2}$ We have checked that the inclusion of $h_{b}, h_{\tau}$ in the calculation does not modify essentially our results. The same occurs if we do the running from $M_{G U T}$ instead of $M_{S U}$.
} 
which corresponds to a range of the top Yukawa coupling $h_{t}$ at the string scale between 0.14 and 0.18 . The lower bound comes from the present experimental limit, whereas the upper bound is due to the sneutrino mass which becomes negative for $m_{t}>105$ $\mathrm{GeV}$ (see fig.1b). The values of $\omega$ can be read off from the top of the plots and they vary in the range between 10.6 and 12.9. Fig.1a shows squark $(\tilde{q})$ and gluino $(\tilde{g})$ masses. In our approximation $\mathrm{u}$ and d-type squarks are degenerate for the first two generations (separately for each chirality and charge), and the right sbottom is also degenerate with the first two right d-squarks. Left and right stops have a non-trivial mixing in their mass matrix which gives rise to the eigenstates $\tilde{t}_{\ell}$ and $\tilde{t}_{h}$. Fig.1b shows Higgs, slepton $(\tilde{\ell})$ and excited photon $\left(\gamma^{*}\right)$ masses. In the usual notation $H^{ \pm}$is the charged Higgs, $A$ is the pseudoscalar, and $H, h$ are the neutral scalars. Again in our approximation, all sleptons are degenerate (separately for each chirality and charge). Fig.1c shows the neutralino $\left(\chi^{o}\right)$ and chargino $\left(\chi^{ \pm}\right)$masses. Note that the masses of sneutrinos, right selectrons, lightest chargino and the two lightest neutralinos are close to their present experimental bounds [19]. The lightest supersymmetric particle (LSP) is a neutralino for $m_{t}<102 \mathrm{GeV}$ and the sneutrino for larger values. Finally, the values of the dynamically generated scale $Q_{0}$ defined in (4.7) are in the range 100-700 GeV, justifying the reliability of the RGE treatment.

The boundary conditions of case 2 in (4.4) lead to a heavy top in the range:

$$
140 \mathrm{GeV} \lesssim m_{t} \lesssim 155 \mathrm{GeV}
$$

which corresponds to a range of the top Yukawa coupling $h_{t}$ at the string scale between 0.4 and 0.38 . The lower bound in (6.12) comes from the present experimental limits on supersymmetric Higgs detection: for $m_{t}<140 \mathrm{GeV}$ the lightest neutral Higgs $h$ becomes too light as shown in fig.2b. Note that radiative corrections to its mass are important in this case, and amount to adding around $20 \mathrm{GeV}$ to the tree-level values [26]. At the upper bound of $m_{t}$ the value of $\mu$ in (6.9) goes to infinity, and beyond it becomes imaginary. However, in this region one should take into account the decoupling of heavy particles in the RGEs to obtain a more reliable result. The values of $\omega$ are now in the range between 1.7 and 4, while $Q_{0}$ varies between 140 and 
$100 \mathrm{GeV}$. In this case the LSP is a neutralino in the whole range of allowed $m_{t}$.

In conclusion, we have presented the phenomenology of the minimal supersymmetric Standard Model in the context of a class of 4D strings with spontaneously broken supersymmetry by a large internal dimension. The scales of supersymmetry breaking and weak interactions are proportional to a unique dynamically determined scale hierarchically smaller than the Planck mass, and the whole spectrum was given as a function of one free parameter, the top quark mass. The boundary conditions at the string unification scale were determined by the supersymmetry breaking mechanism applied in a particular class of compactifications which include the $\mathbf{Z}_{4}$ orbifold. The classification of other possible boundary conditions, arising from different exact discrete symmetries used to break supersymmetry, is an interesting question. On the other hand, the explicit construction of a "realistic" string model exhibiting the required properties remains an open problem.

\section{A Appendix}

We collect here the renormalization group equations for the couplings and soft breaking parameters of the minimal supersymmetric Standard Model. We neglect all Yukawa couplings except those of the third generation.

- Gauge couplings

$$
\frac{d g_{i}^{2}}{d t}=-\frac{b_{i}}{(4 \pi)^{2}} g_{i}^{4}
$$

- Gaugino masses

$$
\frac{d M_{i}}{d t}=-\frac{b_{i}}{(4 \pi)^{2}} g_{i}^{2} M_{i}^{2}
$$

where $t \equiv \ln \left(M_{G U T}^{2} / Q^{2}\right)$ and $b_{3}=-3, b_{2}=1$ and $b_{1}=11$.

- Yukawa couplings of the third generation

$$
\frac{d Y_{t}}{d t}=Y_{t}\left(\frac{16}{3} \tilde{\alpha}_{3}+3 \tilde{\alpha}_{2}+\frac{13}{9} \tilde{\alpha}_{1}-6 Y_{t}-Y_{b}\right)
$$




$$
\begin{gathered}
\frac{d Y_{b}}{d t}=Y_{b}\left(\frac{16}{3} \tilde{\alpha}_{3}+3 \tilde{\alpha}_{2}+\frac{7}{9} \tilde{\alpha}_{1}-Y_{t}-6 Y_{b}-Y_{\tau}\right) \\
\frac{d Y_{\tau}}{d t}=Y_{\tau}\left(3 \tilde{\alpha}_{2}+3 \tilde{\alpha}_{1}-3 Y_{b}-4 Y_{\tau}\right)
\end{gathered}
$$

where one defines

$$
Y \equiv \frac{h^{2}}{(4 \pi)^{2}} ; \quad \tilde{\alpha}_{i} \equiv \frac{\alpha_{i}}{4 \pi}
$$

and the gauge coupling constants at $M_{G U T}$ verify

$$
\alpha_{3}(0)=\alpha_{2}(0)=\frac{5}{3} \alpha_{1}(0)=\alpha_{G U T}
$$

- Trilinear soft terms corresponding to the third generation Yukawa couplings

$$
\begin{gathered}
\frac{d A_{t}}{d t}=\left(\frac{16}{3} \tilde{\alpha}_{3} M_{3}+3 \tilde{\alpha}_{2} M_{2}+\frac{13}{9} \tilde{\alpha}_{1} M_{1}\right)-6 Y_{t} A_{t}-Y_{b} A_{b} \\
\frac{d A_{b}}{d t}=\left(\frac{16}{3} \tilde{\alpha}_{3} M_{3}+3 \tilde{\alpha}_{2} M_{2}+\frac{7}{9} \tilde{\alpha}_{1} M_{1}\right)-Y_{t} A_{t}-6 Y_{b} A_{b}-Y_{\tau} A_{\tau} \\
\frac{d A_{\tau}}{d t}=\left(3 \tilde{\alpha}_{2} M_{2}+3 \tilde{\alpha}_{1} M_{1}\right)-3 Y_{b} A_{b}-4 Y_{\tau} A_{\tau}
\end{gathered}
$$

- Supersymmetric mass of the higgsinos

$$
\frac{d \mu^{2}}{d t}=\left(3 \tilde{\alpha}_{2}+\tilde{\alpha}_{1}-3 Y_{t}-3 Y_{b}-Y_{\tau}\right) \mu^{2}
$$

- Scalar masses

$$
\begin{aligned}
\frac{d m_{Q}^{2}}{d t} & =\left(\frac{16}{3} \tilde{\alpha}_{3} M_{3}^{2}+3 \tilde{\alpha}_{2} M_{2}^{2}+\frac{1}{9} \tilde{\alpha}_{1} M_{1}^{2}\right)-Y_{t}\left(m_{Q}^{2}+m_{u}^{2}+m_{2}^{2}+A_{t}^{2}-\mu^{2}\right) \\
& -Y_{b}\left(m_{Q}^{2}+m_{d}^{2}+m_{1}^{2}+A_{b}^{2}-\mu^{2}\right)-\frac{1}{6} \tilde{\alpha}_{1} S
\end{aligned}
$$




$$
\begin{gathered}
\frac{d m_{u}^{2}}{d t}=\left(\frac{16}{3} \tilde{\alpha}_{3} M_{3}^{2}+\frac{16}{9} \tilde{\alpha}_{1} M_{1}^{2}\right)-2 Y_{t}\left(m_{Q}^{2}+m_{u}^{2}+m_{2}^{2}+A_{t}^{2}-\mu^{2}\right)+\frac{2}{3} \tilde{\alpha}_{1} S \\
\frac{d m_{d}^{2}}{d t}=\left(\frac{16}{3} \tilde{\alpha}_{3} M_{3}^{2}+\frac{4}{9} \tilde{\alpha}_{1} M_{1}^{2}\right)-2 Y_{b}\left(m_{Q}^{2}+m_{d}^{2}+m_{1}^{2}+A_{b}^{2}-\mu^{2}\right)-\frac{1}{3} \tilde{\alpha}_{1} S \\
\frac{d m_{L}^{2}}{d t}=\left(3 \tilde{\alpha}_{2} M_{2}^{2}+\tilde{\alpha}_{1} M_{1}^{2}\right)-Y_{\tau}\left(m_{L}^{2}+m_{e}^{2}+m_{1}^{2}+A_{\tau}^{2}-\mu^{2}\right)+\frac{1}{2} \tilde{\alpha}_{1} S \\
\frac{d m_{e}^{2}}{d t}=\left(4 \tilde{\alpha}_{1} M_{1}^{2}\right)-2 Y_{\tau}\left(m_{L}^{2}+m_{e}^{2}+m_{1}^{2}+A_{\tau}^{2}-\mu^{2}\right)-\tilde{\alpha}_{1} S
\end{gathered}
$$

For the scalars of the first two generations the same equations apply with $Y_{t}=$ $Y_{b}=Y_{\tau}=0$.

- Higgs doublets mass parameters

$$
\begin{gathered}
\frac{d m_{1}^{2}}{d t}=\left(3 \tilde{\alpha}_{2} M_{2}^{2}+\tilde{\alpha}_{1} M_{1}^{2}\right)+\left(3 \tilde{\alpha}_{2}+\tilde{\alpha}_{1}\right) \mu^{2}-3 Y_{t} \mu^{2}-3 Y_{b}\left(m_{Q}^{2}+m_{d}^{2}+m_{1}^{2}+A_{b}^{2}\right) \\
-Y_{\tau}\left(m_{L}^{2}+m_{e}^{2}+m_{1}^{2}+A_{\tau}^{2}\right)+\frac{1}{2} \tilde{\alpha}_{1} S \\
\frac{d m_{2}^{2}}{d t}=\left(3 \tilde{\alpha}_{2} M_{2}^{2}+\tilde{\alpha}_{1} M_{1}^{2}\right)+\left(3 \tilde{\alpha}_{2}+\tilde{\alpha}_{1}\right) \mu^{2} \\
-\left(3 Y_{b}+Y_{\tau}\right) \mu^{2}-3 Y_{t}\left(m_{Q}^{2}+m_{u}^{2}+m_{2}^{2}+A_{t}^{2}\right)-\frac{1}{2} \tilde{\alpha}_{1} S \\
\frac{d m_{3}^{2}}{d t}=\left(\frac{3}{2} \tilde{\alpha}_{2}+\frac{1}{2} \tilde{\alpha}_{1}-\frac{3}{2} Y_{t}-\frac{3}{2} Y_{b}-\frac{1}{2} Y_{\tau}\right) m_{3}^{2} \\
-\left(3 \tilde{\alpha}_{2} M_{2}+\tilde{\alpha}_{1} M_{1}\right) \mu+\left(3 Y_{t} A_{t}+3 Y_{b} A_{b}+Y_{\tau} A_{\tau}\right) \mu
\end{gathered}
$$

where the value of $\mathrm{S}$ is given by

$$
S=m_{2}^{2}-m_{1}^{2}+\sum_{\text {generations }}\left(m_{Q}^{2}+m_{d}^{2}-2 m_{u}^{2}-m_{L}^{2}+m_{e}^{2}\right)
$$




\section{References}

[1] T. Banks and L. Dixon, Nucl. Phys. B307 (1988) 93.

[2] I. Antoniadis, C. Bachas, D. Lewellen and T. Tomaras, Phys. Lett. 207B (1988) 441.

[3] C. Kounnas and M. Porrati, Nucl. Phys. B310 (1988) 355; S. Ferrara, C. Kounnas, M. Porrati and F. Zwirner, Nucl. Phys. B318 (1989) 75; C. Kounnas and B. Rostand, Nucl. Phys. B341 (1990) 641.

[4] I. Antoniadis, Phys. Lett. 246B (1990) 377; Proc. PASCOS-91 Symposium, Boston 1991 (World Scientific, Singapore) p.718.

[5] E.Cremmer, S. Ferrara, C. Kounnas and D.V. Nanopoulos, Phys. Lett. B133 (1983) 61; J. Ellis, A.B. Lahanas, D.V. Nanopoulos and K. Tamvakis, Phys. Lett. B134 (1984) 429; J. Ellis, C. Kounnas and D.V. Nanopoulos, Nucl. Phys. B247 (1984) 373; A.B. Lahanas and D.V. Nanopoulos, Phys. Rep 145 (1987) 1.

[6] J. Scherk and J.H. Schwarz, Phys. Lett. B82 (1979) 60.

[7] P. Fayet, Phys. Lett. B159 (1985) 121; Nucl. Phys. B263 (1986) 649.

[8] R. Rohm, Nucl. Phys. B237 (1984) 553.

[9] J.-P. Derendinger, L.E. Ibáñez and H.P. Nilles, Phys. Lett. 155B (1985) 65; M. Dine, R. Rohm, N. Seiberg and E. Witten, Phys. Lett. 156B (1985) 55.

[10] L. Dixon, J. Harvey, C. Vafa and E. Witten, Nucl. Phys. B261 (1985) 678; Nucl. Phys. B274 (1986) 285.

[11] T.R. Taylor and G. Veneziano, Phys. Lett. 212B (1988) 147.

[12] L. Dixon, V.S. Kaplunovsky and J. Louis, Nucl. Phys. B355 (1991) 649; I. Antoniadis, K.S. Narain and T.R. Taylor, Phys. Lett. B267 (1991) 37.

[13] H. Itoyama and T.R. Taylor, Phys. Lett. 186B (1987) 129. 
[14] For a recent review, see: L.E. Ibáñez and G.G. Ross, CERN preprint CERNTH.6412/92 (1992), to appear in Perspectives in Higgs Physics, ed. G. Kane, and references therein.

[15] N.V. Krasnikov, Phys. Lett. 193B (1987) 37; L. Dixon, talk presented at the A.P.S D.P.F. Meeting at Houston (1990); V. Kaplunovsky, talk presented at the String 90 workshop at College Station (1990); J.A. Casas, Z. Lalak, C. Muñoz and G.G. Ross, Nucl. Phys. B347 (1990) 243; B. de Carlos, J.A. Casas and C. Muñoz, CERN preprint CERN-TH.6436/92.

[16] M. Dine, N. Seiberg and E. Witten, Nucl. Phys. B289 (1987) 589.

[17] M. Cvetic, Proc. Int. Workshop on Superstrings, Maryland 1987 and 1988 (World Scientific, Singapore).

[18] L. Dixon, D. Friedan, E. Martinec and S.H. Shenker, Nucl. Phys. B282 (1987) 13.

[19] Particle Data Group, Review of Particle Properties, Phys. Rev. D45 (1992) S1.

[20] V.A. Kostelecky and S. Samuel, Phys. Lett. B270 (1991) 21.

[21] A. Giveon and M. Porrati, Phys. Lett. B246 (1990) 54; Nucl. Phys. B355 (1991) 422.

[22] K. Inoue, A. Kakuto, H. Komatsu and S. Takeshita, Prog. Theor. Phys. 68 (1982) 927 and Erratum preprint KYUSHU-83-HE-5 (1983); L. Ibáñez, Nucl. Phys. B218 (1983) 514; L. Ibáñez and C. López, Phys. Lett. B126 (1983) 54 and Nucl. Phys. B233 (1984) 511; J. Ellis, J.S. Hagelin, D.V. Nanopoulos and K. Tamvakis, Phys. Lett. B125 (1983) 275; C. Kounnas, A.B. Lahanas, D.V. Nanopoulos and M. Quirós, Phys. Lett. B132 (1983) 95 and Nucl. Phys. B236 (1984) 438; B. Gato, J. León, J. Pérez Mercader and M. Quirós, Nucl. Phys. B253 (1985) 285; N.K. Falck, Z. Phys. C30 (1986) 247. 
[23] L. Alvarez-Gaumé, J. Polchinski and M.B. Wise, Nucl. Phys. B221 (1983) 495; J.P. Derendinger and C. Savoy, Nucl. Phys. B237 (1984) 307.

[24] L. Ibáñez, C. López and C. Muñoz, Nucl. Phys. B256 (1985) 218.

[25] V. Kaplunovsky, Nucl. Phys. B307 (1988) 145 and Erratum preprint StanfordITP-838 (1992).

[26] H.E. Haber and R. Hempfling, Phys. Rev. Lett. 66 (1991) 1815; Y. Okada, M. Yamaguchi and T. Yanagida, Prog. Theor. Phys. 85 (1991) 1 and Phys. Lett. B262 (1991) 54; J. Ellis, G. Ridolfi and F. Zwirner, Phys. Lett. B257 (1991) 83 and B262 (1991) 477; J.R. Espinosa and M. Quirós, Phys. Lett. B266 (1991) 389.

\section{Figure captions}

Fig.1: Masses of the different particles versus $m_{t}$ for the case 1: a) Squarks and gluinos; b) Higgses, sleptons and excited photon; c) Neutralinos and charginos.

Fig.2: The same as in fig.1 but for the case 2 . 